\title{
An Ideal Spin Filter: Long-range, High Spin Selectivity in Chiral Helicoidal 3-Dimensional Metal Organic Frameworks
}

Uxua Huizi-Rayo ${ }^{\mathrm{a}}$, Junkal Gutierrez ${ }^{\mathrm{b}, \mathrm{c}}$, Jose Manuel Seco ${ }^{\mathrm{a}}$, Vladimiro Mujica ${ }^{\mathrm{de}, \mathrm{e}}$,* $^{*}$ Ismael Diez-Perez ${ }^{\mathrm{g}}$, Jesus M. Ugalde ${ }^{\mathrm{f}, *}$, Agnieszka Tercjak $^{\mathrm{b}}$, Javier Cepeda ${ }^{\mathrm{a}}$ and Eider San Sebastian ${ }^{\mathrm{a},{ }^{*}}$

a Departamento de Química Aplicada, Facultad de Química, Universidad del País Vasco (UPV/EHU), 20018 Donostia, Spain. E-mail: eider.sansebastian@ehu.eus

b Group 'Materials+Technologies' (GMT), Department of Chemical and Environmental Engineering, Faculty of Engineering Gipuzkoa, University of the Basque Country (UPV/EHU), Plaza Europa 1, 20018, DonostiaSan Sebastian, Spain

${ }^{c}$ Department of Chemical and Environmental Engineering, Faculty of Engineering Vitoria-Gasteiz, University of the Basque Country (UPV/EHU), C/ Nieves Cano 12, 01006, Vitoria-Gasteiz, Spain

d Arizona State University, School of Molecular Sciences, Tempe, AZ 85287, U.S.A. E-mail: vmujica@asu.edu

e Ikerbasque, Basque Foundation for Science, 48011 Bilbao, Spain

${ }^{\mathrm{f}}$ Kimika Fakultatea, Euskal Herriko Unibertsitatea and Donostia International Physics Center (DIPC), P. K. 1072, 20080 Donostia, Euskadi, Spain.E-mail: jesus.ugalde@ehu.eus

${ }^{g}$ Department of Chemistry, Faculty of Natural \& Mathematical Sciences, King's College London, Britannia House, 7 Trinity Street, London SE1 1DB, UK

\section{S1.- Chiroptical characterization.}

S2.- Diffuse reflectance experiments and band-gap estimation.

S3.- Dimensional analysis of Dy-L single crystals by AFM.

S4. -Optical characterization of Dy-L single crystals.

S5.- Charge transport measurements.

S6.- Conductivity estimations. 


\section{S1.-Chiroptical characterization.}

$\mathrm{CD}$ (and the corresponding UV-Vis) spectra were reported previously by our group and are reproduced below. The CD spectra were acquired for a water suspension of Dy-L (and Dy-D) as well as for the water solutions of L- and D- tartaric acid samples. As observed in Figure S1 CD spectra of the MOFs are characterized by two well-defined regions: an intense band centered at $195 \mathrm{~nm}$ and a less intense one at $221 \mathrm{~nm},{ }^{1}$ with opposite Cotton effects, respectively. Bands at the same wavelengths are of course present in the corresponding UVVis spectra and can be assigned to $\mathrm{n} \rightarrow \pi^{*}$ transitions according to TD-DFT calculations. CD spectra of enantiomeric samples are mirror images, clearly indicating the enantiopurity of the samples. These results are in agreement with those reported by Tushari et al. for compounds Er-L and Er-D. ${ }^{2}$ Crucially, CD spectra of water solutions of L- and D-tartaric acids (free ligands) show two bands at 190 and $214 \mathrm{~nm}$ which are blueshifted and inverted (+/- sign) compared to the ones observed for the MOFs samples.
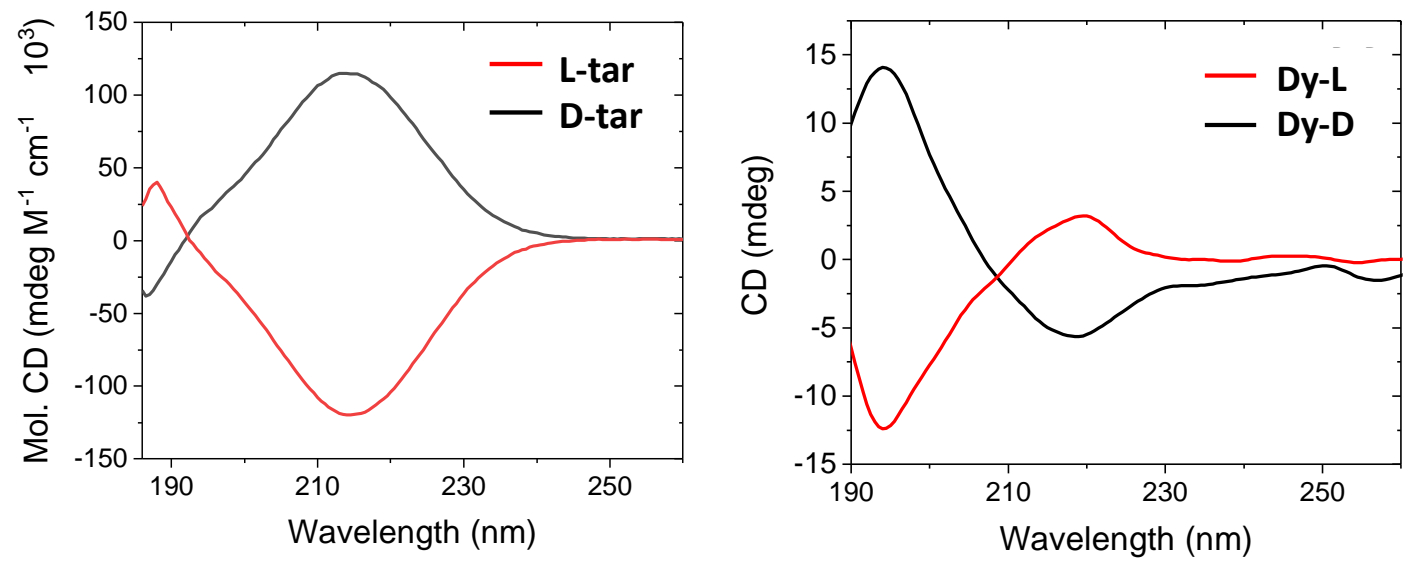

Figure S1. CD spectra of water solutions of L-tar and D-tar and water suspensions of Dy-L and Dy-D.

\section{S2.- Diffuse reflectance experiments and band-gap estimation.}

Diffuse reflectance spectrum on a pressed pellet of single crystals of Dy-L was acquired with a Shimadzu UV2600 spectrometer in the $190-900 \mathrm{~nm}$ at room temperature, using barium sulphate as reflectance standard. (Figure S2). The spectrum revealed the expected electronic transitions of different nature, the least energetic $\mathrm{f} \rightarrow \mathrm{f}$ transition being located in the NIR region, at ca. $808 \mathrm{~nm}(1.53 \mathrm{eV})$. According to the literature,$^{3}$ less energetic transitions are generally found at ca. $1488 \mathrm{~nm}$, which confirms that Dy-L are low band gap materials with an estimated value of $0.77 \mathrm{eV}$, which falls below the range of typical semiconductor materials such as GaAs $1.4 \mathrm{eV}), \mathrm{Si}(1.2 \mathrm{eV})$ and $\mathrm{Ge}(1.7 \mathrm{eV})$.

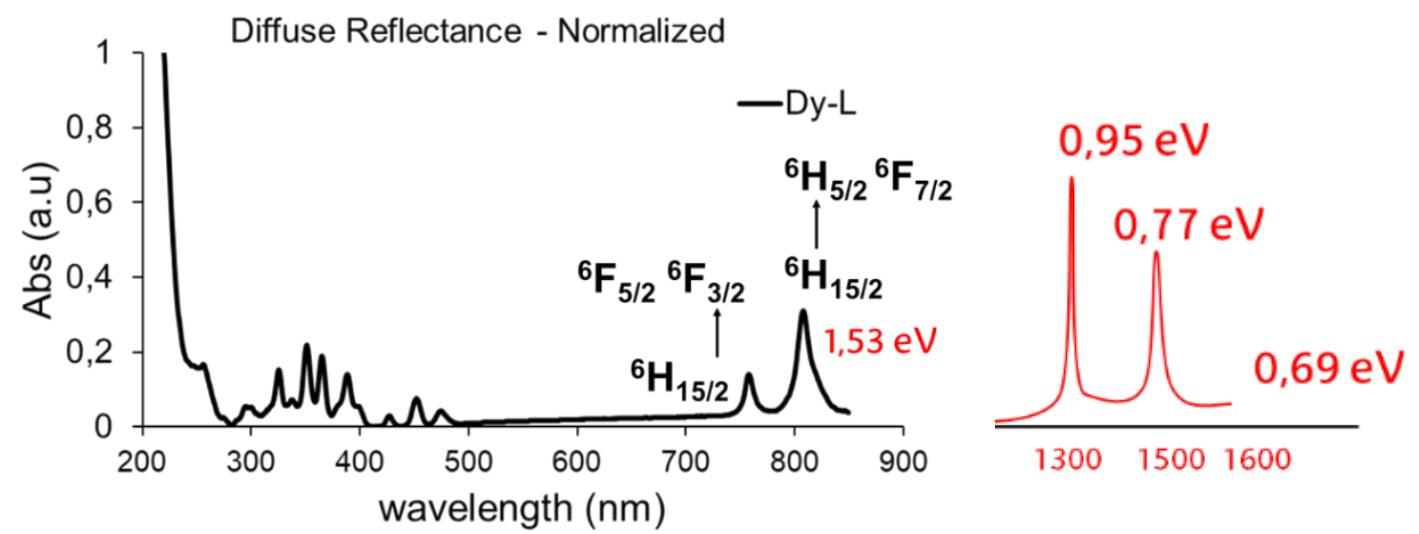

Figure S2. Normalized diffuse reflectance spectrum of pressed pellets of Dy-L single crystals (black). Additional transitions reported. ${ }^{4}$ 


\section{S3.- Dimensional analysis of Dy-L single crystals by AFM}

As shown in the Figure S3, AFM height image confirmed the dimensional characteristics of analyzed Dy-L single crystals. AFM height image profiles showed that the height of these analyzed single crystals was $\sim 200 \mathrm{~nm}$ while the relation of 2D dimensions was equal to $0.8 \mu \mathrm{m} / 1 \mu \mathrm{m}$.
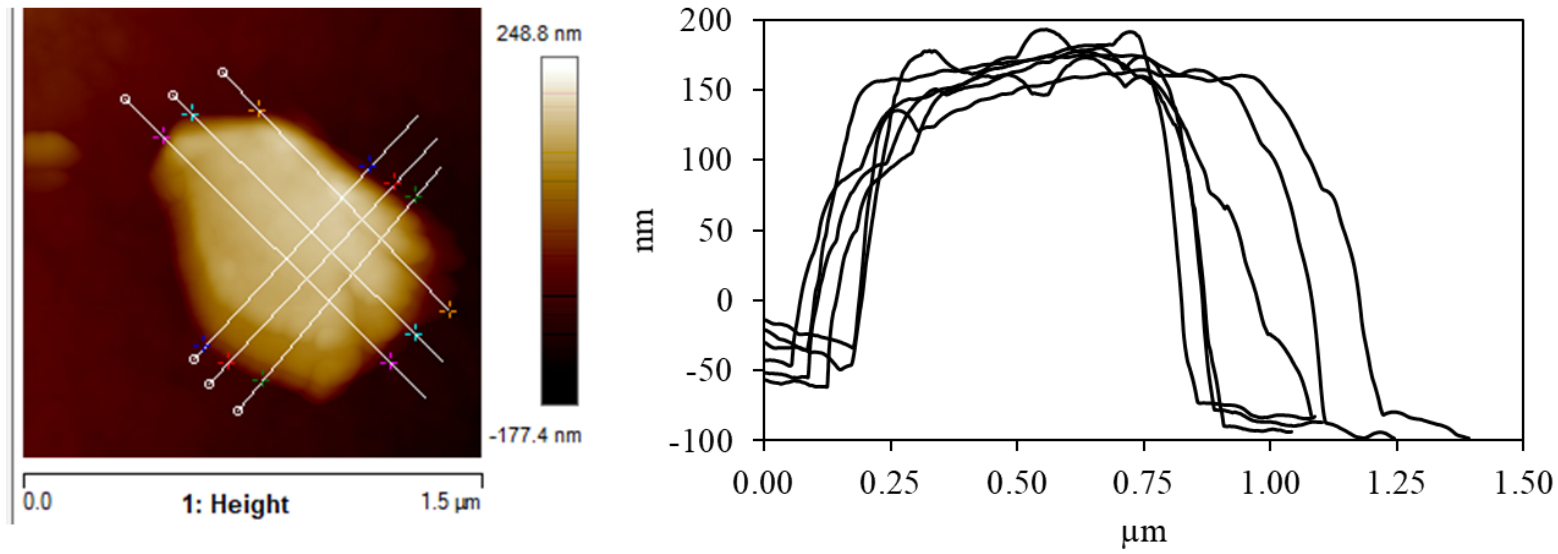

Figure S3. AFM dimensional analysis of Dy-L single crystal and corresponding profiles.

\section{S4. Optical characterization of Dy-L single crystals.}

Optical micrographs proved the presence of single crystals over ITO surface. Although the diversity in size is notable, the prismatic shape is quite defined and homogeneous (Figure S4a and b), in addition to the fact that all samples have the transparency and brightness that characterize single crystals. It should be noted that, as seen in Figures S4 c and d, among the largest crystals, others of nanometric dimensions can be seen that may be more suitable for AFM measurements.
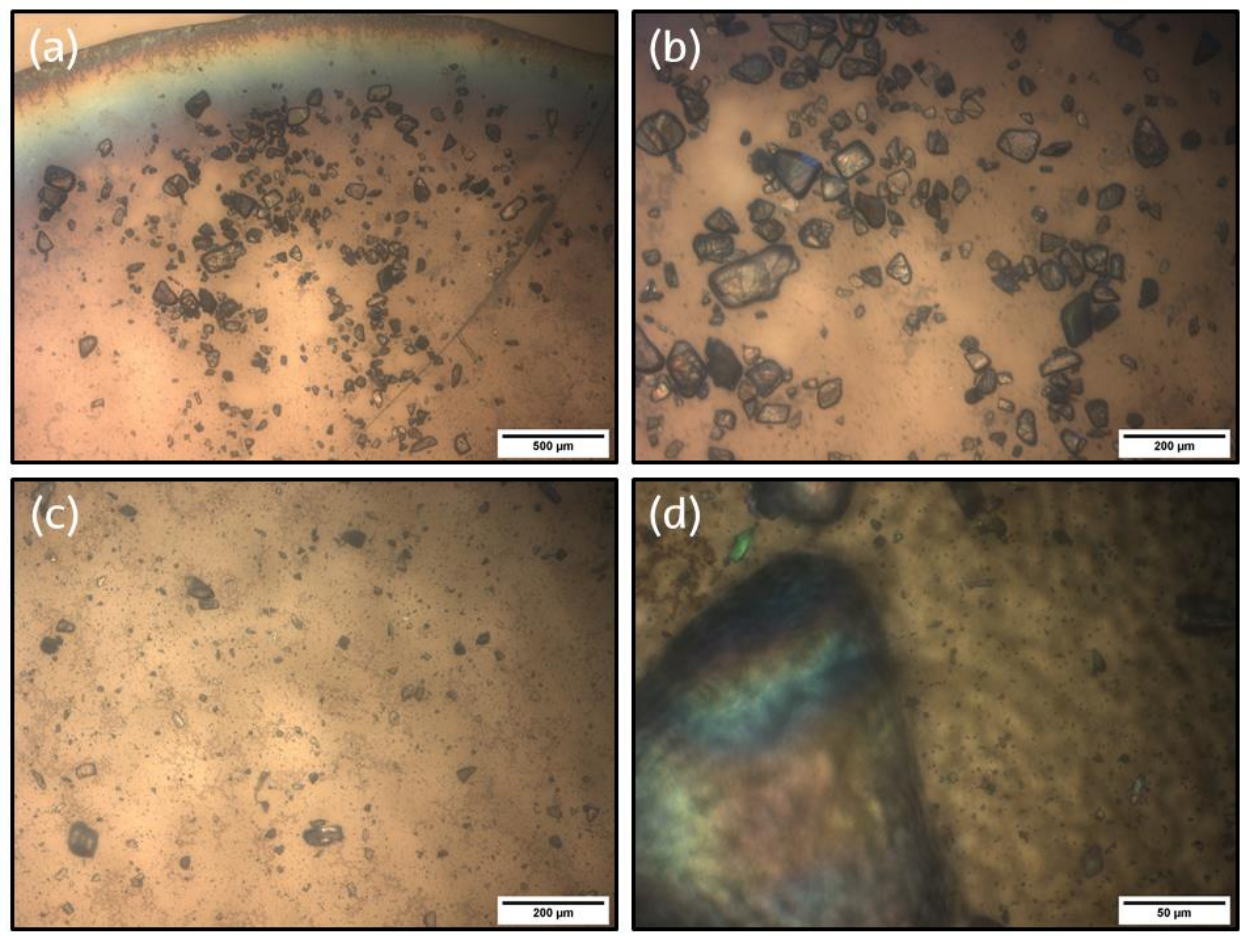

Figure S4. Observation of single crystals by optical microscope. (a) Single crystals of different sizes over ITO surface. (b) Amplification in the observed area that shows the homogeneity of crystals. (c) Observation of a different area with smaller crystals. (d) Amplification of a new observed area that shows the smallest single crystals. 
Indexation of the faces of the single crystals was done according to the analogy of their shape using the crystal exposed to X-ray as a reference. As observed in the photographs in Figure S4 crystal exhibits a main (quite planar) face that corresponds to $\left(\begin{array}{lll}0 & 0 & 1\end{array}\right)$ Miller index (shown in the front view). Axis containing the latter reflection (index) is displayed in the second image, taken by turning the crystal $90^{\circ}$ to the right. Last image shows the first view but incorporating $\left(\begin{array}{lll}-1 & 0 & 0\end{array}\right)$ index. This fact, allows us concluding that crystallographic $c$ axis crosses the main face of the crystal, whereas $a$ and $b$ axes are almost perpendicular to it.

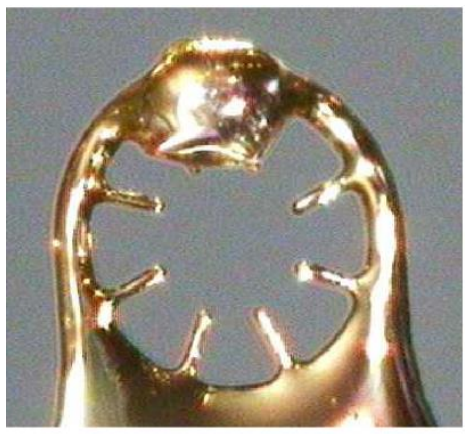

Front view

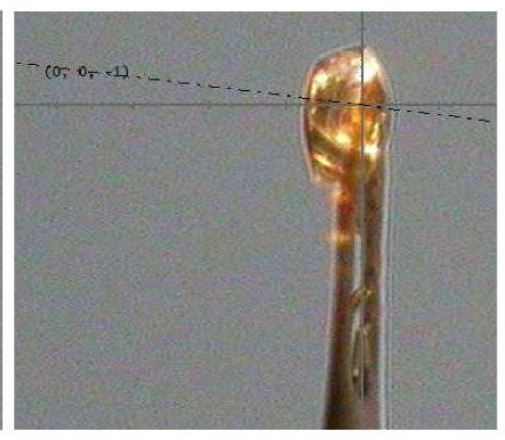

Turn of $90^{\circ}$

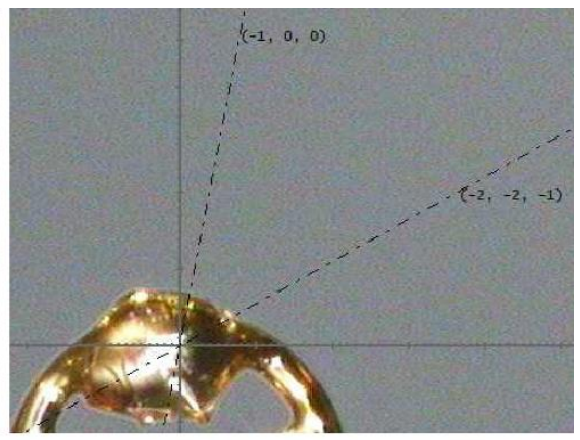

Front view showing $\left(\begin{array}{lll}-1 & 0 & 0\end{array}\right)$ face

Figure S5. Photographs of single crystals taken on the diffractometer, showing detail of the crystal faces (h k 1).

\section{S5.- Charge transport measurements}

For charge transport measurements, current-voltage (I/V) curves were measured for Dy-L at room temperature. Through statistical analysis, the averages of $\mathrm{Tip}_{0}$, Tip + and Tip. curves were obtained, including the standard deviation for each case. I/V curves for Dy-L single crystals deposited on ITO substrate were recorded in the full $\pm 10 \mathrm{~V}$ bias range for the $\mathrm{Tip}_{0}$, Tip. and Tip + tip magnetic polarizations. Experiments were run in both ramping directions, i.e. from - to + bias values and from + to - bias values, respectively; experiments in each ramping direction was run 50 times $(n=50)$. In these cases, electrical hysteresis is observed, derived from the electrical polarization of the charges in the Dy-L single crystals upon current transmission, which was still present upon the two minutes gap left before running the experiment in the second voltage ramping direction. Also, were indicated, $n=100 \mathrm{I} / \mathrm{V}$ curves ( $\mathrm{n}=50$ in each ramping directions) were averaged over both ramping directions, thus, neglecting the hysteresis effect.

Experiments were run, were indicated (Tip + set-up), with two alternative amplifiers with the aim of getting not saturated I/V curves. The sharp signal saturation initially observed in the case of Tip magnetizations of the AFM probe was alleviated within a fairly large voltage range of $\pm 2 \mathrm{~V}$, where the current could be measured and the I/V characteristics displayed the expected linear behavior flanked by two exponential (non-linear) branches, i.e., I/V curves that exhibit the S-shape behavior characteristic of molecular junctions and solar cells, as opposed to the abrupt jump in current in Figure S6 and S7 c) and d). Still, due to the high current levels flowing across the MOF crystal in this particular magnetization direction, our current amplifiers saturated again beyond this voltage range, but, whereas Figure S6 and S7 c) and d) show Tip signals saturated at ca. 200pA, Figures S6 and S7 c') and d') now saturate at ca. 1-2 nA, i.e. it seems that large current crossing our single crystals in the $\mathrm{Tip}_{+}$experiments, exceeds the limits of our amplifier again.

Figure S7 is nearly the same as Figure S6 but with I/V curves as a function of applied bias in the $\pm 6 \mathrm{~V}$ range averaged over 50 measurements, and obtained either in the - to + ramping direction (solid lines) or + to ramping direction (dashed lines), where hysteresis is observed; a) and b) results obtained in the Tip 0 setup, c) and d) and e) and f), as in a) and b) but magnetizing the Tip either in the + or - arbitrary directions, respectively. $c^{\prime}$ ) and $d^{\prime}$ ), as in c) and d) but with an alternative amplifier that permitted the measurement of higher currents. 
$I / V$ curves $(-10 \mathrm{~V}$ to $+10 \mathrm{~V})$
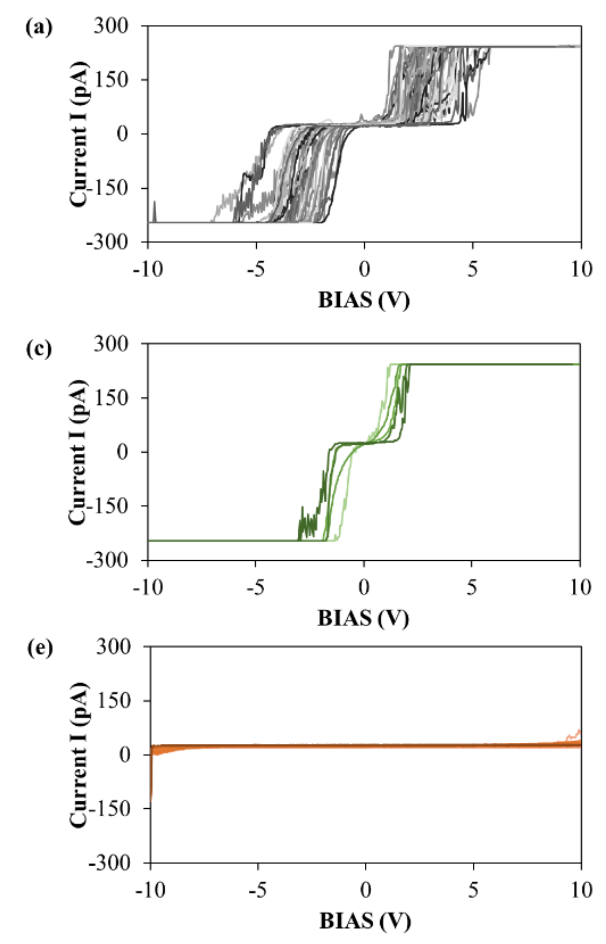

$\mathrm{I} / \mathrm{V}$ curves (+10 V to -10 V)

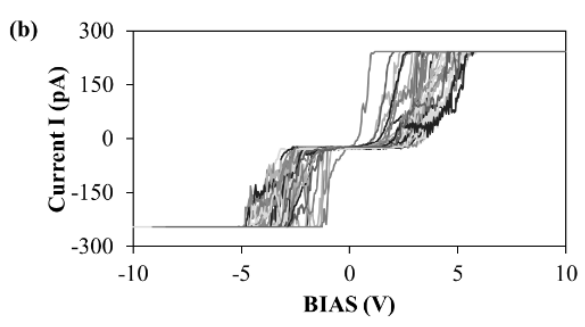

(d)
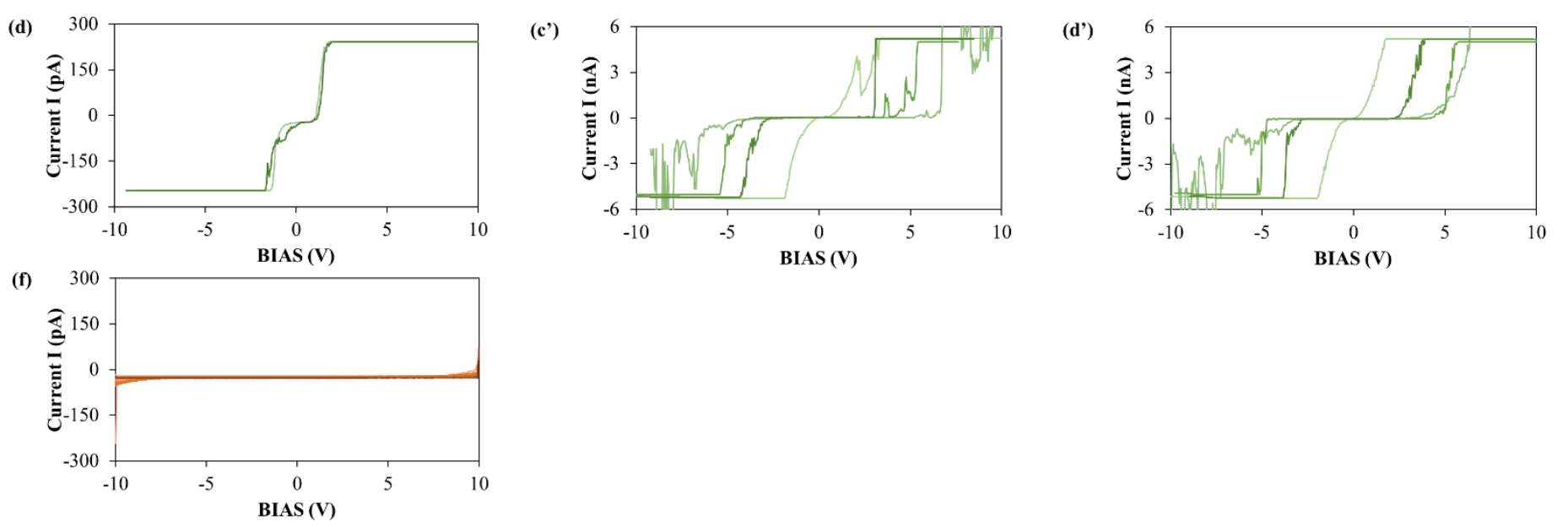

Figure S6. I/V curves recorded in the full $\pm 10 \mathrm{~V}$ bias range for Dy-L single crystals deposited on ITO substrate. (a) and (b) are results from the Tip 0 experimental set-up with no AFM tip magnetization: (a) Individual ( $\mathrm{n}=50$ ) experiments with voltage ramping from negative to positive values; (b) Individual experiments ( $\mathrm{n}=50$ ) with voltage ramping from positive to negative values. (c) and (d), as in (a) and (b), respectively, but with tip magnetization arbitrarily north (Tip $p_{+}$experimental set up); $\mathrm{c}^{\prime}$ ) and d'), as in c) and d) but with an alternative amplifier that permitted the measurement of higher currents $(\mathrm{n}=4)$. (e) and (f), as in (a) and (b), respectively, but with tip magnetization arbitrarily south (Tip- experimental set up). 
For charge transport measurements, current-voltage (I/V) curves were measured for Dy-L at room temperature. Through statistical analysis, the averages of Tip ${ }_{0}$, Tip ${ }_{+}$and Tipcurves were obtained, including the standard deviation (SD) for each case (shown as grey bars in Figure S7).

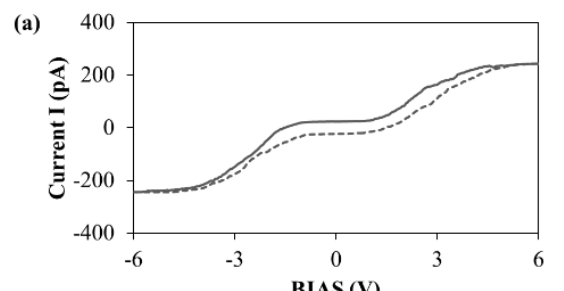

BIAS (V)
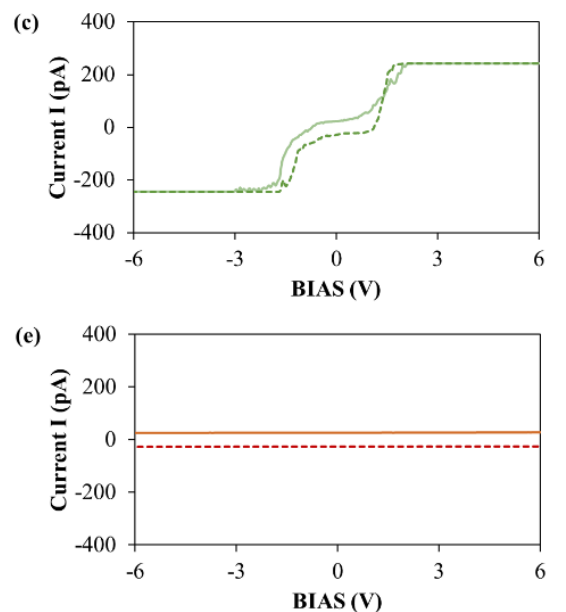

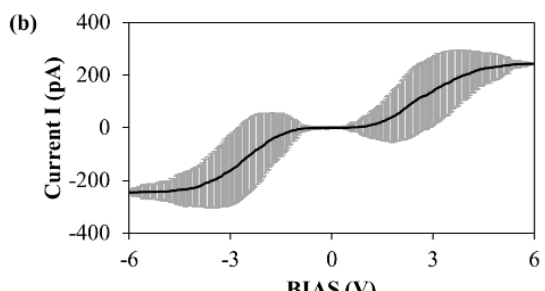

BIAS (V)
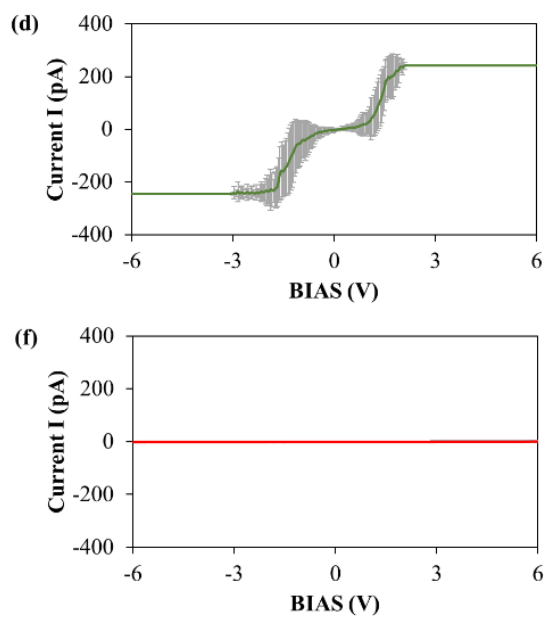
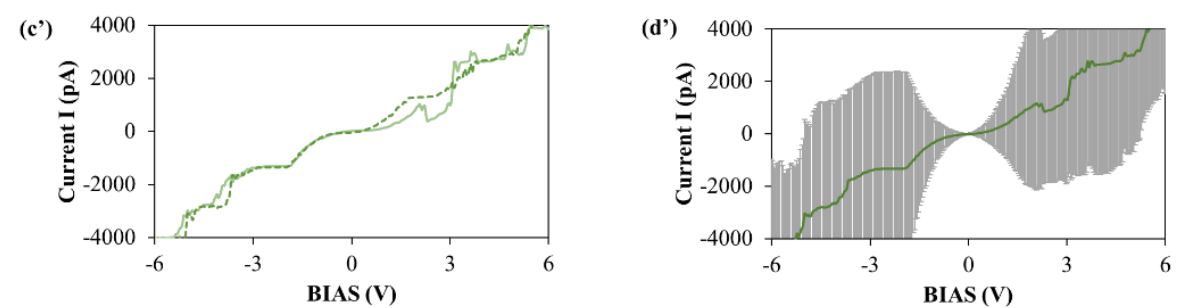

Figure S7. Same as Figure S6 but with $\mathrm{I} / \mathrm{V}$ curves as a function of applied bias in the $\pm 6 \mathrm{~V}$ range averaged over 50 measurements, and obtained either in the - to + ramping direction (solid lines) or + to - ramping direction (dashed lines), where hysteresis is observed; a) nd b) results obtained in the Tip0 setup, c) and d) and e) and f), as in a) and b) but magnetizing the tip either in the + or - arbitrary directions, respectively. $c^{\prime}$ ) and $d^{\prime}$ ), as in c) and d) but with an alternative amplifier that permitted the measurement of higher currents. Grey bars represent SD values. 


\section{S7.- Conductivity estimations}

As it is a non-ohmic material (relationship between the applied voltage and the resulting electric current is not linear), the method of static resistance was applied to estimate conductivity in arbitrary linear regions using equation 1, equation 2 and equation 3, where $X, R, \rho, A, L$ and $\sigma$ represent the slope of the curve, the resistance of the material, the resistivity of the material, the cross-sectional area of a crystal, the length of the crystal and the conductivity of the material, respectively.

$$
\begin{aligned}
& R=\frac{1}{X} \text { equation } 1 \\
& \rho=R \frac{A}{L} \text { equation } 2 \\
& \sigma=\frac{1}{\rho} \text { equation } 3
\end{aligned}
$$

Homogeneity of single crystals was confirmed by AFM morphology images. As Figure S8 shows, A and L were calculated according to the height and dimensions given by AFM height image profiles (see Figure S3), obtaining values of $10^{-8} \mathrm{~cm}$ and $2 \times 10^{-5} \mathrm{~cm}$, respectively.
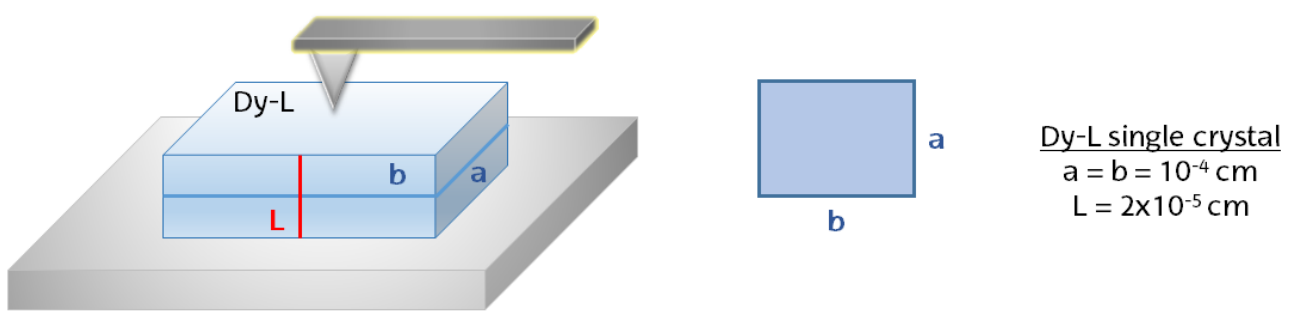

Figure S8. Set-up of conductance measurements in single crystals deposited on ITO substrate, and single crystal dimensions: Section $\mathrm{A}=\mathrm{a} * \mathrm{~b}$ and height $\mathrm{L}$.

In table $S 1$, conductance values for the $\pm 1 \mathrm{~V}$ linear regions in the $\mathrm{I} / \mathrm{V}$ curves of the $\mathrm{Tip}_{0}, \mathrm{Tip}_{+}$and Tipexperimental setups are shown, respectively.

Table S1. Conductivity values $(\mu \mathrm{S} / \mathrm{cm})$ obtained for single crystals of $\mathbf{D y}-\mathbf{L}$ in the three distinct experimental conditions; $\mathrm{SD}=$ Standard Deviation

\begin{tabular}{|c|c|c|c|}
\hline Set up & Bias Range & $\sigma(\mu \mathrm{S} / \mathrm{cm})$ & SD \\
\hline Tip $_{0}$ & $-1.0 \mathrm{~V}$ to $1.0 \mathrm{~V}$ & 0.00645 & \pm 0.00027 \\
\hline Tip $_{+}$ & $-1.0 \mathrm{~V}$ to $1.0 \mathrm{~V}$ & 0.28694 & \pm 0.01020 \\
\hline Tip - & $-1.0 \mathrm{~V}$ to $1.0 \mathrm{~V}$ & 0.00022 & \pm 0.00001 \\
\hline
\end{tabular}

\footnotetext{
${ }^{1}$ Huizi-Rayo,U.; Zabala-Lekuona,A.; Terenzi, A.; Cruz, C.M.; Cuerva, J. M.; Rodríguez-Diéguez, A.; García, J. A.; Seco, J. M.; San Sebastian, E.; Cepeda, J. Influence of Thermally-Induced Structural Transformations over Magnetic and Luminiscence Properties of Tartrate-based Chiral Lanthanide Organic-Frameworks. J. Mat.Chem. C 2020,8, 8243-8256..

${ }^{2}$ Thushari, S.; Cha, J. A. K.; Sung, H. H.-Y.; Chui, S. S.-Y.; Leung, A. L.-F.; Yen, Y.-F.; Williams, I. D. Microporous chiral metal coordination polymers: hydrothermal synthesis, channel engineering and stability of lanthanide tartrates. Chem. Commun. 2005, 5515-5517.

${ }^{3}$ Volkovich, V. A.; Ivanov, A. B.; Yakimov, S. M.; Tsarevskii, D. V.; Golovanova, O. A.; Sukhikh, V. V.; Griffiths, T. R. Electronic Absorption Spectra of Rare Earth (III) Species in NaCl-2CsCl Eutectic Based Melts. Physics, Technologies and Innovation (PTI-2016). AIP Conf. Proc. 1767, 020023-1-020023-11.
} 